\title{
APROXIMACIONES A LA FONOLOGÍA DEL CHEDUNGUN: UN ESTUDIO EXPLORATORIO EN NIÑOS PEWENCHES DEL ALTO BÍO-BÍO ${ }^{1}$ \\ PHONOLOGY APPROACHES TO CHEDUNGUN: AN EXPLORATORY STUDY IN PEWENCHES CHILDREN FROM ALTO BÍO-BÍO.
}

\author{
Dra. Marisol Henríquez Barahona ${ }^{2}$ \\ Universidad Católica de la Santísima Concepción, Chile \\ mhenriquez@ucsc.cl
}

\begin{abstract}
Resumen:
El propósito de este artículo es examinar de manera preliminar la fonología del chedungun en niños bilingües del Valle del Queuco, en Alto Bío-Bío. Concretamente nos hemos propuesto abordar algunos rasgos fonético-fonológicos prominentes que caracterizan el sistema fonológico de estos niños en relación con las descripciones fonológicas existentes del pewenche hablado por adultos de la zona.

Para el desarrollo de este estudio, trabajamos con un grupo de 20 niños bilingües, cuyas edades oscilan entre los 10 y 14 años, quienes pertenecen a las comunidades de Callaqui, Pitril, TrapaTrapa y Butalelbun. Para la recogida de datos fonético-fonológicos utilizamos la re-narración de una historia breve en chedungun y una lista léxica de 100 palabras.

En términos generales, los datos muestran la ocurrencia de algunos rasgos característicos de las comunidades en estudio que no necesariamente comparten todos los niños. Se observa un alto grado de mantenimiento de la fonología tradicional del chedungun en los hablantes de las comunidades de Butalelbun y Trapa-Trapa, mientras que en los sectores de Callaqui y Pitril, se observan innovaciones y sustituciones atribuibles a los diversos grados de bilingüismo que presentan los niños de estos sectores.
\end{abstract}

Palabras claves: Fonología del chedungun- Niños bilingües- Alto Bío-Bío-Bilingüismo.

Abstract: The aim of this paper is to carry out a preliminary research on chedungun phonology in bilingual children from Queuco Valley, Alto Bio-Bio. The goal we have set is to address some prominent phonetic-phonological features that characterize the phonological system of these children based on the phonological descriptions of pewenche spoken by adults.

For the purpose of this study, we worked with a group of 20 bilingual children. They belong to the communities of Callaqui, Pitril, Trapa-Trapa and Butalelbun and their ages range from 10 to 14 . For phonetic-phonological data collection a retelling of a short story in chedungun and a lexical list of 100 words were used.

Overall, the data shows the occurrence of some typical features of the communities under study, that are not shared by all children. Butalelbun and Trapa-Trapa are the communities where the preservation of chedungun traditional phonology is high; whereas in the communities of Callaqui and Pitril innovations and substitutions are observed in children. This is associated with different levels of bilingualism.

Key words: Chedungun phonology- Bilingual children- Alto Bío Bío- Bilingualism

\footnotetext{
${ }^{1}$ Los resultados que aquí se presentan están asociados al proyecto Fondecyt de Iniciación N N 11150508 : “Contacto mapudungun - castellano: rasgos fonético-fonológicos del castellano presentes en el chedungun de escolares pewenches del Alto Bío-Bío".

${ }^{2}$ Académica adscrita al Centro de Investigación CIBAS de la Universidad Católica de la Santísima Concepción.
} 


\section{Introducción}

El panorama sociolingüístico sobre la situación del mapudungun es bastante heterogéneo. Lo que se constata en las diferencias significativas en la situación que presenta la lengua en función de factores diversos como las diferencias regionales, los distintos segmentos poblacionales, los hablantes urbanos y rurales, etc., incluso, se ha señalado, que al interior de una misma región geográfica se pueden observar divergencias en el comportamiento lingüístico de los sujetos (cf. Vergara y Gundermann, 2005; Zúñiga, 2007; Gundermann et al., 2008, 2009; Henríquez, 2014).

Con respecto a la situación sociolingüística de los más jóvenes, los datos proporcionados por Gundermann y otros, muestran que el grupo etario de entre 10 y 19 años es el que presenta menor desempeño en la lengua mapuche, puesto que sólo un 14,3\% comprueba competencia, concentrándose en los niveles básicos e intermedio y con un importante número de hablantes pasivos (2009). Este grupo se caracteriza por presentar los menores índices de bilingüismo, pero su desempeño no es el mismo en los diferentes territorios.

Para el caso del Alto Bío-Bío, zona que nos ocupa en este estudio, se ha observado un escenario bastante positivo para la lengua con muchos hablantes competentes en todas las edades, sin embargo, es posible observar diferencias en la competencia comunicativa entre los niños dependiendo de su comunidad de procedencia. Lo que tiene directa relación con diversos factores sociolingüísticos producto del contacto sociohistórico. (Henríquez, 2014, 2015).

En este contexto, parece necesario desarrollar estudios focalizados para examinar el estado actual del sistema lingüístico mapuche, en nuestro caso, el sistema fonológico. Esto, porque como se sabe, la restricción funcional de una lengua producto del contacto lingüístico intenso y prolongado provoca variaciones y cambios no solo en la competencia linguiística de sus usuarios, sino que también se manifiesta en el plano formal (Thomason y Kaufman, 1998; Thomason, 2001). De este modo, nos hemos propuesto desarrollar este estudio que tiene como foco identificar y describir algunos rasgos prominentes que caracterizan la 
Marisol Henríquez B. Aproximaciones a la fonología del Chedungun: un estudio exploratorio en niños Pewenches del Alto Bío-Bío

fonología segmental del chedungun de niños bilingües de cuatro comunidades del Valle del río Queuco.

\section{Descripciones de la fonología segmental del mapuche-pewenche o chedungun ${ }^{3}$}

Diversas son las descripciones fonológicas especializadas del mapuche hablado por adultos. De estas, destacamos los trabajos de: Suárez (1959); Echeverría (1964); Salas (1976, 1978); Lagos (1981); Álvarez-Santullano (1986a, 1986b); Sánchez (1989); Salamanca (1997); Salamanca y Quintrileo (2009); Sadowsky, Painequeo, Salamanca y Avelino (2013) y Alvarez-Santullano, Risco y Forno, (2016); entre otros.

Con respecto a la fonología segmental del mapuche-pewenche o chedungun hablado en el Alto Bío-Bío, conocemos la existencia de dos trabajos que contienen inventarios de fonemas, ambos, realizados con hablantes adultos en la zona del valle del Queuco en Alto Bío-Bío: Sánchez (1989) y Salamanca (1997).

La descripción fonológica de Sánchez se realizó con datos recogidos en la comunidad de Cauñicu. El autor propone un inventario de 23 fonemas segmentales, de los cuales 6 son vocales: /a, e, i, o, u, ü/ y 17 son consonantes: 3 oclusivas /p, t, k/; 2 africadas /tr, g/; 6 fricativas /v, ð, s, f, j, w /; 4 nasales /m, n, n, y/ y 2 laterales /1, $\lambda$ /. De acuerdo con Sánchez (1989), los pewenches del Alto Bío-Bío realizan las fricativas labiodental e interdental como sonoras (o ensordecidas) [v] y [ð], pero nunca como sordas. Además, sostiene que en esta variante no habría distinción entre los sonidos alveolares e interdentales (excepto /s/ versus /ð/. Este hecho diferenciaría al pewenche de Alto Bío- Bío, del picunche del llano central y del pewenche meridional. Para Salas, esta supuesta falta de diferenciación entre alveolares e interdentales no es tan clara, especialmente en la localidad de Cauñicu, la misma en que Sánchez recogió sus textos (Salas, 1992a: 510).

Del estudio de Salamanca, quien recoge los datos en la comunidad de Malla Malla en el valle del río Queuco, resulta un inventario de 27 fonemas segmentales: 6 vocales /a, e, i, o,

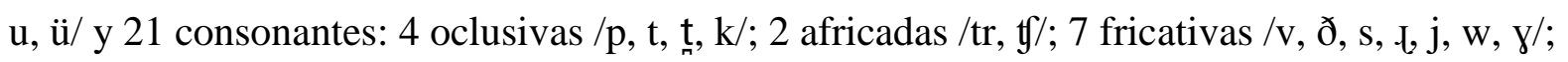
5 nasales $/ \mathrm{m}, \mathrm{n}, \mathrm{n}, \mathrm{n}, \mathrm{y} /$ y 3 laterales $/ 1,1, \lambda /$. Este autor, a diferencia de Sánchez, asigna

\footnotetext{
${ }^{3}$ En este estudio usaremos las denominaciones pewenche y chedungun, para referirnos a la variante pewenche del mapudungun.
} 
estatus fonémico a la fricativa velar $[\mathrm{g}]$ y a los sonidos interdentales $[\mathrm{t}],[\mathrm{n}]$ y $[\mathrm{l}]$, aunque reconoce que en algunos hablantes la distinción entre estos últimos y los sonidos alveolares no es tan regular (1997:120). Con respecto a los fonemas /ð/ y /v/, declara que el fonema fricativo interdental se realiza siempre sonoro, en lo que coincide con Sánchez; en cambio, el fonema fricativo labiodental, además de la variante labiodental sonora [v], presentaría ocasionalmente la realización fricativa labiodental áfona [f]. En esto se distancia de Sánchez.

Para Salamanca, no existirían grandes diferencias entre la fonología del pewenche hablado en Alto Bío-Bío y la del mapuche central de Chile (Salamanca, 1997:123). De igual modo, Salas señala que los pewenches del Alto Bío-Bío "presentan diferencias lingüísticas pequeñas, pero claramente perceptibles, tanto con respecto a los pehuenches más meridionales -o sea, de Malleco, Cautín y Valdivia- como con respecto a la población picunche del llano central y de la costa" (Salas, 1992b: 62).

Para el caso de la población pewenche infantil, Henríquez y Salamanca (2012) describen los rasgos prominentes de la fonología de los escolares de dos comunidades del valle del Queuco: Butalelbun y Cauñicu, encontrando algunas diferencias fonéticofonológicas en los sistemas de estos hablantes, especialmente en los segmentos consonánticos. El inventario de fonemas vocálicos de ambas comunidades no difiere de aquél que se presenta en las descripciones clásicas del mapuche, en general, y del pewenche de Alto Bío-Bío, en particular: /a e, i, o, u, ə/. En cuanto a las consonantes, en Butalelbun se registran 21 fonemas consonánticos: $/ \mathrm{p}, \mathrm{t}, \mathrm{t} \mathrm{k}, \mathrm{t} \int \mathrm{tr}, \mathrm{m}, \mathrm{n}, \mathrm{n}, \mathrm{n}, \mathrm{n}, \mathrm{v}, \mathrm{\gamma}, \mathrm{s}, \mathrm{\gamma}$,

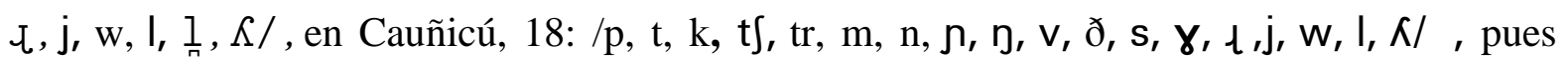
están ausentes las (inter) dentales / $t, n_{n}, I_{n} /$, que, si bien es cierto, se observan en algunos niños de esta comunidad, no ocurren de manera sistemática. Por otra parte, estos autores al abordar la vitalidad de la fonología segmental en las mismas comunidades del Alto Bío-Bío, destacan la alta vitalidad de varios de los segmentos críticos analizados, entre los que sobresalen la "sexta” vocal /ü/(ə); la nasal velar / $/$; la aproximante retrofleja / $/$ / y la africada retrofleja /ts/ los cuales presentan índices de vitalidad de más del $80 \%$ en las palabras efectivamente respondidas (Henríquez y Salamanca, 2015). Entre los fonemas menos vitales están las interdentales $/ t, n_{n}, I_{n} /$, que presentan una baja frecuencia, especialmente en Cauñicu, 
Marisol Henríquez B. Aproximaciones a la fonología del Chedungun: un estudio exploratorio en niños Pewenches del Alto Bío-Bío

donde perdieron el estatus de fonemas. En esta última comunidad, además, se observaron importantes diferencias en el rendimiento de los segmentos analizados, lo que se explica producto de las transferencias fonológicas del castellano que han provocado numerosas alternancias y, a veces, el reemplazo de estos fonemas en algunos escolares (Henríquez, 2013, Henríquez y Salamanca, 2015).

\section{La Investigación}

\subsection{Objetivo}

El objetivo que nos hemos propuesto en este estudio es relevar los principales rasgos fonéticos fonológicos que caracterizan el sistema fonológico del chedungun hablado por niños bilingües de cuatro comunidades del Alto Bío-Bío.

Este trabajo se focalizará en el análisis de los siguientes segmentos: a) el fonema vocálico /ə/ b) los fonemas consonánticos /t, n, l, J, ts, v, t $5 /$. Esto, debido a que son segmentos que han sido objeto de discusión entre los especialistas, ya sea por su estatus dudoso o por su inestabilidad producto de la influencia del español o por ser eventuales marcadores de variación dialectal, por lo que resulta interesante e informativo su abordaje, especialmente en el sistema fonológico de los niños.

\subsection{Los participantes}

La muestra está compuesta por un grupo de 20 niños pewenches, bilingües chedungunespañol, cuyas edades fluctúan entre los 10 y 14 años. Estos colaboradores viven en alguna de las cuatro comunidades seleccionadas: Callaqui, Pitril; Trapa-Trapa y Butalelbun.

Todos los niños participaron de manera voluntaria. Sus padres nos dieron su consentimiento informado para realizar la elicitación de los datos, mientras que los niños, su asentimiento informado.

Todos los colaboradores son bilingües en diferentes grados; algunos poseen competencias productivas más desarrolladas (Butalelbun y Trapa Trapa); otros, demuestran competencias productivas menores. Esto lo pudimos observar con la aplicación de la re-narración de una historia breve en chedungun. En todo caso es necesario señalar que el chedungun que manejan estos niños es el que les han transmitido sus padres y abuelos en el seno familiar. La incidencia de la escuela es menor, pues el Programa EIB y, más específicamente, el subsector de 
Lengua indígena se aplica, por el momento, en los niveles menores de enseñanza (1 a 4 año básico).

\subsection{Estrategias para la recolección del material}

Para la elicitación del corpus fonético-fonológico se utilizaron dos estrategias:

\subsubsection{Aplicación de una lista léxica}

Se utilizó una lista léxica de 100 ítems, compuesta de palabras relacionadas con partes del cuerpo, alimentos, términos de parentesco, flora, fauna, colores, numerales, etc. Al seleccionar la lista de palabras tuvimos especial cuidado en incluir todos los fonemas de la lengua mapuche, especialmente aquellos que son el foco de este estudio: /ə, t, n, 1, ð, v, ts, t $\int /$.

\subsubsection{Narración oral de una historia breve}

En términos generales, diseñamos y presentamos a los niños un relato breve en mapudungun, que contenía palabras seleccionas de la lista léxica. Luego de escuchar la grabación en dos oportunidades, debían narrar la historia en mapudungun o contar lo que recordaban de ella. En los casos en que los escolares tuvieron dificultad para narrar el cuento en mapudungun, o si en su relato no aparecían las palabras seleccionadas, se les realizaron algunas preguntas específicas sobre la historia para lograr la elicitación de éstas.

El texto de la historia fue revisado y grabado por un hablante pewenche de la zona.

3.4. Sistema de transcripción: la transcripción de las respuestas elicitadas se realizó con los símbolos del AFI, para lo cual se realizaron audiciones pasivas y deliberadas de los datos registrados. Para el análisis auditivo, se empleó la grabadora TASCAM DR-40; y para el apoyo visual, se utilizó una cámara HANDYCAM SONY HDR-CX440.

\section{Resultados}

En el siguiente apartado presentaremos los rasgos fonético-fonológicos más prominentes de la fonología del mapuche de los niños de las comunidades de Callaqui, Pitril, Trapa Trapa y Butalelbun. Estos constituyen los primeros hallazgos de nuestro estudio y resultan al comparar estos sistemas fonológicos con aquellos que se presentan en las 
Marisol Henríquez B. Aproximaciones a la fonología del Chedungun: un estudio exploratorio en niños Pewenches del Alto Bío-Bío

descripciones fonemáticas existentes del mapudungun en general y con la variante mapuche pewenche, en particular; es decir, el contraste se realizará principalmente con el sistema fonológico descrito por Sánchez (1989) y Salamanca (1997), para hablantes pewenches adultos del valle del Queuco en Alto Bío-Bío y con las descripciones de Henríquez y Salamanca para la población escolar $(2012,2015)$.

\subsection{Vocal, central media no redondeada /a /}

Con respecto a las vocales nos interesa específicamente la situación de la vocal central media no redondeada /ə/ por ser un segmento vocálico exclusivo del mapudungun o chedungun, es decir, ausente en el inventario de fonemas del español, lo que en una situación de interacción de sistemas podría provocar cierta inestabilidad del segmento, como ha ocurrido en el sistema fonológico de niños de otras zonas como en Tirúa (Henríquez, 2016a, 2016b).

De acuerdo con el análisis del material recolectado en el sector, se ha podido constatar la ocurrencia de la denominada "sexta vocal mapuche" /ə/ en las cuatro comunidades en estudio. Este fonema presenta dos variantes: alta posterior no redondeada [u] y central media no redondeada [ə]. Esta última variante es la que presenta mayor número de ocurrencias entre los niños, por lo que la hemos relevado como la representante del fonema

Ejemplos de ocurrencias:

(1) [kə.'la] "tres" (Pitril)

(2) [wən] "boca” (Trapa-Trapa)

(3) [kə.'tsal] "fuego" (Butalelbun)

(4) [an'tə] "sol/día" (Trapa Trapa)

(5) [km.'tsal] (Callaqui)

(6) [ə.'nəm] (Callaqui).

El análisis de nuestros datos nos permite señalar que este fonema es de uso muy recurrente, pues aparece en la mayoría de las palabras respondidas por los niños en las que 
era expectable su ocurrencia. Observamos, además, algunos procesos de alternancia (7) y reemplazos (8), (9) que se dan en Pitril y Callaqui, aunque de manera más frecuente en esta última comunidad. Estas alternancias o reemplazos ocurren principalmente con el fonema $/ \mathrm{u} /$.

$$
\begin{aligned}
& \text { (7) [leu.'vu] [leu..'vu] “río” (Pitril) } \\
& \text { (8) [ku.'tfal] “fuego/fogón” (Callaqui) } \\
& \text { (9) [an.'tu] “día/sol” (Callaqui). }
\end{aligned}
$$

\section{2 (Inter) Dentales $/ \mathrm{t}, \mathrm{n}_{n}, \mathrm{l} /$}

El estatus de estos fonemas ha sido objeto de discusión entre los especialistas, pues algunos reconocen su existencia, mientras que otros la niegan. Para la variante pewenche adulta, Sánchez (1989) no otorga estatus de fonema a estos segmentos; mientras que Salamanca sí distingue entre alveolares e interdentales (1997), aunque reconoce que esta distinción no es del todo regular. Salas, por su parte, en relación a Cauñicu, comunidad del valle del Queuco, donde Sánchez recogió sus datos, señala que “...en lo que respecta a la separación entre interdentales y alveolares, no hay grandes diferencias en relación a lo que ocurre en el resto de la Araucanía" (1992a), reconociendo así la existencia de estos fonemas. Para el caso de los niños, Henríquez y Salamanca, en un estudio realizado con escolares de las localidades de Cauñicu y Butalelbun, relevan el estatus fonémico de $/ \mathbf{t}, \mathbf{n}, \mathbf{n} /$ en Butalelbun, pero no en Cauñicu, donde los niños presentan realizaciones esporádicas de estos fonos con abundantes procesos de transferencia y reemplazo $(2012,2015)$.

Ahora bien, según el análisis de nuestro corpus, observamos realizaciones sistemáticas de las interdentales en las comunidades de Butalelbun y Trapa Trapa, aunque en menor medida en esta última comunidad. Efectivamente, las realizaciones interdentales se producen consistentemente en la mayoría de las palabras realizadas por los niños en las que era expectable su ocurrencia (2) (4), (10), (11). No sucede lo mismo en Pitril, donde se observan las realizaciones dentales, pero de manera asistemática, es decir, se observan realizaciones, pero también procesos de alternancia y reemplazo por sus homólogas, las alveolares /t, n, 1/: (12), (13). 
La situación de estos segmentos en la comunidad de Callaqui es muy diferente a lo observado en las otras localidades, pues no hemos constatado ninguna realización de estos fonos, ya que en todas las palabras realizadas por los niños en las que era expectable su ocurrencia, fueron sistemáticamente reemplazadas por las alveolares [t], [n], [1]: (14), (15)

Ejemplos:

(10) [tta'pəl] “'hoja de árbol” (Butalelbun)

(11) [pun] "noche" (Butalelbun y Trapa-Trapa)

(12) $[$ pun] $~[$ pun] “noche" (Pitril)

(13) [ta.'pul] [ta.'pul] "hoja de árbol” (Pitril)

(14) [tol] "frente" (Pitril)

(15) [an.'tə] “día/sol” (16) [na.'mun] "pie/pata”

A continuación presentamos, a modo de ejemplo, dos imágenes (archivos de videos), donde podemos observar claramente la realización de la interdental nasal [n] en posición final de palabra (Fig. 1) y en posición inicial (Fig. 2)

Figura 1: [wun] "boca” (niño de Butalelbun, 12 años)

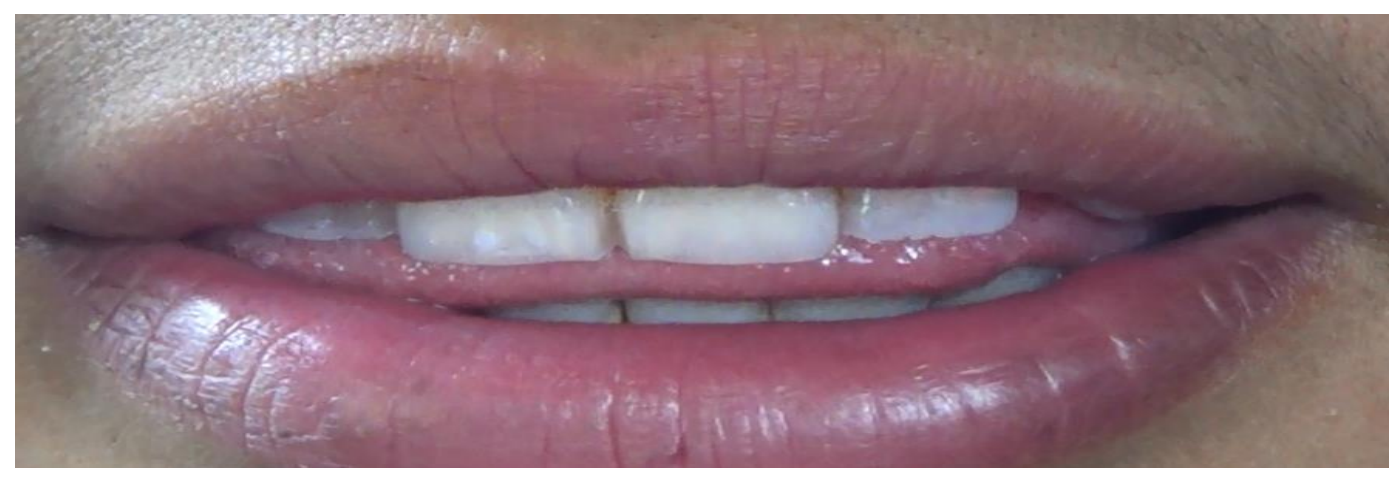

Figura 2: [na'mun] "pie/pata” (niña de Butalelbun, 13 años). 


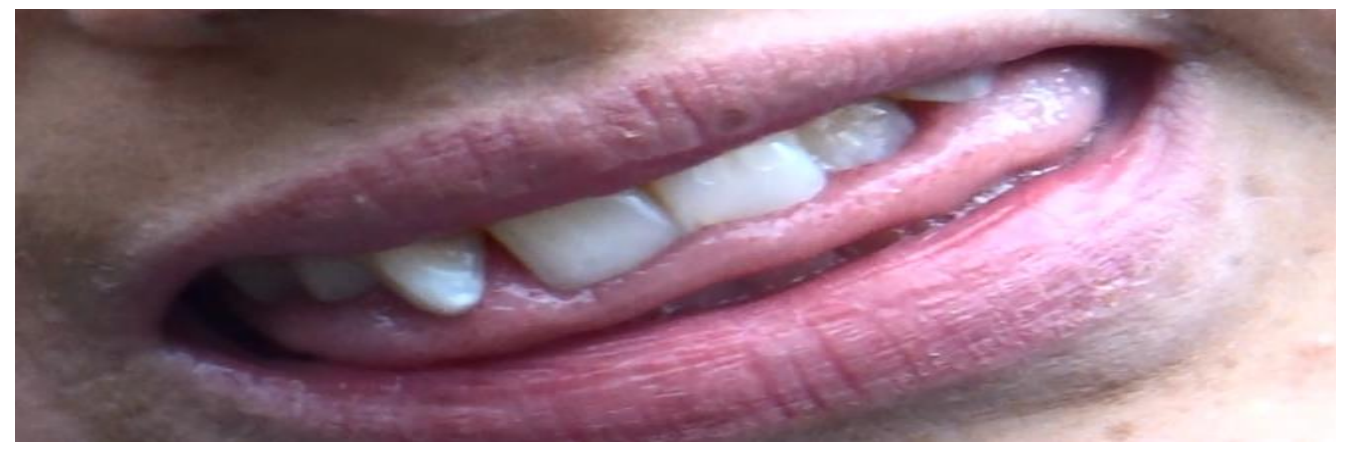

Finalmente, es necesario consignar que los fonemas dentales $/ \mathbf{t}, \mathbf{n}_{n}, \mathbf{l} /$ forman parte del inventario de fonemas del mapudungun, pero no del castellano; por lo que es factible hipotetizar algún grado de desestabilización o innovación, explicable por la influencia de la lengua dominante. En este contexto, la ocurrencia de estos segmentos en la fonología de niños pewenches como los de Butalelbun, resulta muy alentador, ya que constituye según, nuestro entender, un claro indicador de la vitalidad tradicional del sistema en esa zona.

\subsection{Fricativas labiodental sonora $/ v /$ y fricativa interdental sonora //}

Como ya se señaló, según Sánchez los pewenches del Alto Bío-Bío realizan las fricativas labiodental e interdental como sonoras (o ensordecidas) [v] y [ð] pero nunca como sordas $[\theta]$ y [f]. Salamanca, por su parte, destaca la sonorizacion total de [ð], en lo que coincide con Sánchez, pero reconoce la ocurrencia ocasional de [f]. Por su parte, Salas señala con respecto a esta variante "En mi experiencia, predominan las sonoras, pero se escuchan ocasionalmente, realizaciones definitivamente sordas" (1992a:510).

Efectivamente, de acuerdo con el análisis fonológico de nuestros datos, y en concordancia con Salas (1992a), constatamos la existencia de ambas realizaciones de estos fonemas, pero con un claro predominio de las realizaciones sonoras [v] y [ð] por sobre las áfonas $[\theta]$ y [f]. Con respecto al fonema /v/, podemos comentar lo siguiente:

a) Según nuestro análisis, el alófono [v] es la forma básica del fonema, porque es la realización de mayor frecuencia entre los niños. (16), (17), (18).

b) Hemos observado que las realizaciones áfonas [f] ocurren en variación libre con [v], aunque predominan en el postmargen silábico. (19), (20) y (21). 
Marisol Henríquez B. Aproximaciones a la fonología del Chedungun: un estudio exploratorio en niños Pewenches del Alto Bío-Bío

c) Las realizaciones áfonas las hemos observado en las cuatro comunidades.

Ejemplos:

$$
\begin{aligned}
& \text { (16) [və.'ta] "marido/viejo" } \\
& \text { (17) [moK.'və̃] "sangre" } \\
& \text { (18) [vu.'ti] “espalda” } \\
& \text { (19) [liv] [lif] "blanco/limpio" } \\
& \text { (20) [lav.'k'en] [laf.'kjen] "mar/lago" } \\
& \text { (21) [tuf.kjen] [tuv.k'jen] "ceniza" }
\end{aligned}
$$

Con respecto al fonema /ð/, en este primer acercamiento a la fonología del chedungun de los niños, podemos informar lo siguiente:

a) Según nuestro corpus, el alófono sonoro [ð] es la forma básica del fonema, porque no solo es la variante más recurrente entre los niños, sino la más extendida; pues, a diferencia de $[\theta]$, está presente en las cuatro comunidades en estudio y en todos los colaboradores.

b) Las escasas realizaciones de [0] ocurren en alternancia con [ð]. (22) y (23).

c) Tanto en Callaqui como en Pitril, no reportamos realizaciones de [ $\theta$ ]; es decir, en las palabras realizadas en las que era expectable la ocurrencia de uno u otro fono, solo ocurrió la sonora [ð]. (24) y (25).

Ejemplos:

$$
\begin{aligned}
& \text { (22) }[\text { t } \mathrm{o} \theta] \sim\left[\mathrm{t} \int \mathrm{o}\right] \text { "amarillo" }
\end{aligned}
$$

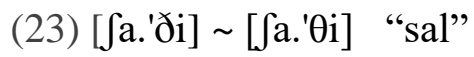

$$
\begin{aligned}
& \text { (24) [ma.wi.'ða] "montaña” } \\
& \text { (25) [pu.'ðo] "ombligo" }
\end{aligned}
$$

Por último, es interesante señalar que hemos registrado algunas realizaciones oclusivas del fono [ð]. Un ejemplo de ello es la ocurrencia de [t $\int \mathrm{a}$. 'di] "sal" realizada por un niño de Butalelbun. Estos casos son aislados, pero los hemos registrado, pues nos informan de 
ciertas innovaciones (Escobar y Wölck, 2009) presentes en el habla de algunos niños bilingües, producto del contacto de sistemas.

\subsection{Realizaciones del fonema africado retroflejo sordo}

De acuerdo con las descripciones con foco en el nivel fonológico del chedungun hablado en la zona (Salamanca, 1997; Henríquez y Salamanca, 2012), el fonema /ts / tiene dos alófonos: uno africado retroflejo sordo [ts] y otro oclusivo retroflejo sordo [t].

Nuestros datos muestran que ambos alófonos están presentes en la zona, pero que, por lejos, el africado es el más recurrente entre los colaboradores y ocurre en todas las comunidades, mientras que la variante con prominencia oclusiva presenta una realización menor entre los niños y, según nuestro corpus, no se registra en Callaqui. Ambos alófonos ocurren en variación libre en la zona (26), (27), (28) y (29).

Ejemplos:

$$
\begin{aligned}
& \text { (26) [te.'wă]- [tse.'wa] "perro" } \\
& \text { (27) [lava.'ta] [lava.'tsa] "rana" } \\
& \text { (28) [wen.'tsu] [wen.'tu] "hombre" } \\
& \text { (29) [ku.'tal] [ku. 'tsal] "fogón/fuego" }
\end{aligned}
$$

Además de las realizaciones anteriores, también registramos algunas ocurrencias castellanizadas del fonema /ts /. Estas se observaron cuando algunos niños realizaron /tse.'wa/ y /wen.' tsu/ como (30) [tre.'wa] y (31) [wen.'tru]. Estas innovaciones son escasas y se reportan principalmente en Callaqui.

\subsection{Realizaciones del fonema africado alveopalatal sordo /ty/}

Según nuestro corpus, este fonema presenta dos alófonos en la zona: uno africado alveopalatal sordo [t] y otro fricativo alveopalatal sordo [f]. De estas dos variantes la más frecuente es la africada, razón por la cual la hemos escogido como la representante del fonema. Sin embargo, es posible constatar, aunque en menor medida, la ocurrencia de la 
Marisol Henríquez B. Aproximaciones a la fonología del Chedungun: un estudio exploratorio en niños Pewenches del Alto Bío-Bío

fricativa en todas las comunidades. Varios son los ejemplos en que estos alófonos alternan en variación libre entre los niños (32), (33), (34) y (35).

Ejemplos:

$$
\begin{aligned}
& \text { (32) [a.tfa.'wal] - [a.fa.'wal] "gallina" } \\
& \text { (33) [tfal.tu.'mai] - [ } \text { al.tu.'mai] "gracias" } \\
& \text { (34) [t fa.'ði] - [ [a.'ði] (Pitril) "sal" } \\
& \text { (35) [do.mo.'fe] - [ðo.mo.'tfe] “mujer” }
\end{aligned}
$$

Ahora bien, según la revisión de la literatura, tanto Sánchez (1989) como Salamanca (1997), no observaron la realización del fono [S] en el chedungun de los adultos del Alto BíoBío. Sin embargo, Henríquez y Salamanca (2012) dan cuenta de la existencia de este fono entre los niños de las comunidades de Cauñicu y Butalelbun, aunque reportan escasas ocurrencias. En este contexto, sería relevante realizar descripciones actuales de la fonología de los adultos del Valle del Queuco para verificar la ocurrencia de la fricativa, pues son estos hablantes quienes, fundamentalmente, transmiten la lengua a los niños, por lo que es muy probable su ocurrencia entre ellos.

Finalmente, desde un punto de vista dialectal, es relevante constatar el funcionamiento de [S] como alófono de $/ \mathrm{t} \int /$, pues en otras variantes del mapudungun este fono fricativo se ha reportado como un alófono de /s/ (Salas, 1976) o como un fonema diferente (Sadowsky, Painequeo, Salamanca y Avelino, 2013; Henríquez, 2016a).

\section{Reflexiones finales}

En términos generales, los datos muestran la ocurrencia de algunos rasgos fonéticofonológicos característicos de las comunidades en estudio que emergen al comparar el sistema fonológico de los niños con el de los adultos de la zona (Sánchez, 1989 y Salamanca, 1997). Entre estos rasgos podemos relevar los siguientes: 
- De acuerdo a nuestros datos, destacamos la ocurrencia de las consonantes (inter)dentales [t, $\mathrm{n}_{n}$ y $\left.\prod_{n}\right]$ entre los niños de las comunidades de Butalebun, Trapa-Trapa y Pitril. En Callaqui no observamos ninguna de estas realizaciones. La ocurrencia de este segmento se destaca principalmente en la comunidad de Butalelbun. Así, concordamos en este análisis con lo reportado por Henríquez y Salamanca, (2012 y 2015), para los niños del sector y con lo propuesto por Salamanca, (1997) para la población adulta de Malla Malla.

- Constatamos la ocurrencia del fonema central medio no redondeado / a / en todas las comunidades, aunque con menor frecuencia en Pitril y Callqui. De las dos realizaciones de este segmento [u] y [ə ], hemos relevado el alófono central como la forma básica del fonema pues es el de mayor frecuencia entre los niños.

- Con respecto las fricativas labiodental /v/ e interdental /ð/ observamos la ocurrencia de ambas realizaciones de estos fonemas, pero con un claro predominio de las sonoras [v] y [ð] por sobre las áfonas $[\theta]$ y [f]. De estas últimas, la menos frecuente es la interdental áfona $[\theta]$, que incluso no ocurre en todas las comunidades.

- Por último, es interesante señalar que en nuestro corpus hemos evidenciado procesos de alternancia y sustitución de fonemas. Estas innovaciones ocurren más frecuentemente en las Comunidades de Pitril y Callaqui y tienen directa relación con los segmentos (inter)dentales [t, n y I], con la sexta vocal [ə], con la africada retrofleja [ts], entre otros. Creemos que esto es atribuible a los diversos grados de bilingüismo que presentan los niños de estos sectores. En estas comunidades los adultos son quienes fundamentalmente hablan la lengua. En el caso de los niños, el panorama es diferente, pues estos son bilingües pero con menor nivel de competencia productiva que lo observado, por ejemplo en niños de Butalelbun y TrapaTrapa.

\section{Bibliografía}

Alvarez-Santullano, Pilar. Descripción fonológica del Huilliche, un dialecto del mapuche o araucano del centro-sur de Chile. Tesis para optar al grado de Magister en Artes con Mención en Lingüística. Universidad de Concepción. (1986a). Impreso.

Alvarez-Santullano, Pilar. "Descripción fonemática del Huilliche: estudio comparativo". Alpha 2, (1986b):45-50.Impreso. 
Marisol Henríquez B. Aproximaciones a la fonología del Chedungun: un estudio exploratorio en niños Pewenches del Alto Bío-Bío

Alvarez-Santullano, Risco, E. y Forno, A. "Descripción fonético-fonológica del sistema consonántico del mapuche hablado en territorio huilliche en los albores del siglo XXI: a propósito de la noción de continuum”. RLA, 54 (2016): 101-127. Impreso.

Echeverría, Max. "Descripción fonológica del mapuche actual". Boletín del Instituto de Filología de la Universidad de Chile, 16 (1964):13-59. Impreso.

Escobar, Ana María y Wölck, Wolfang (Eds.) Contacto lingüístico y la emergencia de variantes y variedades lingüísticas. Vervuert: Iberoamericana, 2009. Impreso.

Gundermann, H., Canihuan, J., Clavería, E. y Faúndez, A. (2008). Perfil sociolingüístico de comunidades mapuches de la Región del Biobío, Araucanía, los Ríos y los Lagos. Informe de Investigación. Santiago: CONADI - UTEM. (2008). Impreso.

---------. "Permanencia y desplazamiento, hipótesis acerca de la vitalidad del mapuzugun". RLA, 47(2009): 37-60. Impreso.

Henríquez, Marisol. Vitalidad fonológica del mapudungun en escolares mapuches pewenches y lafkenches de la VIII región del Bío-Bío. Tesis de Doctorado en Linguiística. Universidad de Concepción, 2013.Impreso.

--------. "Estado del mapudungun en comunidades pewenches y lafkenches de la región del Bío-Bío: El caso de los escolares”. RLA, 52 (2014): 13-40.Impreso.

."Ámbitos de uso del mapudungun en comunidades pewenches y lafkenches de la región del Bío-Bío”. Literatura y Lingüística, 31 (2015): 185-204.Impreso.

----------."Estado de la fonología segmental del mapudungun de escolares lafkenches de la comuna de Tirúa: rasgos prominentes”. Literatura y Lingüística, 34 (2016a):295-318. Impreso.

----------. "Vitalidad de la fonología segmental del mapudungun de escolares de Tirúa". Nueva Revista del Pacífico, 64 (2016b), 27-66. Impreso.

Henríquez, Marisol y Gastón, Salamanca. "Vitalidad de la fonología segmental del chedungun hablado por escolares del Alto Bío-Bío”. Alpha, 41 (2015): 207231.Impreso

--------.'"Rasgos prominentes de la fonología segmental del chedungun hablado por escolares pewenches del Alto Bío-Bío". Alpha, 34 (2012):152-172.Impreso.

Lagos, Daniel. "El estrato fónico del mapudungu(n)". Nueva Revista del Pacífico 19-20 (1981):42-66. Impreso.

Sadowsky, Scott; Héctor, Painequeo; Gastón Salamanca y Heriberto, Avelino. "Mapudungun". Journal of the International Phonetic Association, 43(2013):87-96. Impreso.

Salas, Adalberto. "Esbozo fonológico del mapudungu, lengua de los mapuches o araucanos de Chile central". Estudios Filológicos, 11(1976):143-153. Impreso.

."Mapuche-Español. Análisis fonológico contrastivo". VICUS. CuadernosLingüística, 2(1978):57-85. Impreso.

-------. “Lingüística mapuche. Guía bibliográfica”. Revista Andina, 10 (1992a): 473-537. Impreso.

--------. El mapuche o araucano de Chile. Fonología, gramática y antología de cuentos. Madrid: Fundación MAPFRE América. (1992b). Impreso.

Salamanca, Gastón. "Fonemas segmentales del pewenche hablado en el Alto Bío- Bío". RLA, 35(1997):113-124. Impreso.

Salamanca, Gastón y Elizabeth Quintrileo. "Fonemas segmentales y muestra lexical del mapudungun hablado en Tirúa". RLA, 47(2009):13-35. Impreso. 
Sánchez, Gilberto. "Relatos orales en pewenche chileno". Anales de la Universidad de Chile, 17 (1989):289-360. Impreso.

Suárez, Jorge. "The Phonemes of an Araucanian Dialect". International Journal of American Linguistics, 25 (1959):177-181. Impreso.

Thomason, Sarah. Language contact. Edinburgh: University Press 2001. Impreso.

Thomason, Sarah y Terrence, Kaufman. Language contact, creolization, and genetic linguistics. Bekerley/Los Angeles: University of California Press, 1988. Impreso.

Vergara, Jorge Ivan y Gundermann, Hans (2005): Descripción del contexto sociolingüístico en comunidades indígenas de Chile. Santiago: Programa EIB MINEDUC/ Programa Orígenes. Impreso.

Zúñiga, Fernando. "Maudunguwelaymi am? ¿Acaso ya no hablas mapudungun? Acerca del estado actual de la lengua mapuche”. Centro de Estudios Públicos, 105 (2007):9-24. Impreso. 